\title{
A ATIVIDADE DO MÉDICO ULTRA-SONOGRAFISTA APRESENTA RISCOS PARA O SISTEMA MÚSCULO-ESOUELÉTICO?*
}

\author{
Letícia Holtz Barbosa ${ }^{1}$, Helenice Jane Cote Gil Coury ${ }^{2}$
}

Resumo OBJETIVO: O objetivo deste estudo foi realizar um levantamento de aspectos pessoais, ocupacionais e de sintomas músculo-esqueléticos percebidos por médicos ultra-sonografistas. MATERIAIS E MÉTODOS: Participaram deste estudo 41 médicos ultra-sonografistas que responderam a uma entrevista e a um questionário relacionado a aspectos organizacionais e à percepção de sintomas. Utilizaram-se, para a análise estatística dos resultados, os coeficientes de variação e de Spearman, e o teste $t$ de Student. Análise de regressão logística foi utilizada para identificar a contribuição de algumas variáveis na determinação do desconforto. RESULTADOS: Os resultados mostraram alta taxa de desconforto músculo-esquelético relacionado ao trabalho $(85 \%)$. Entre os sintomáticos, a prevalência de dor foi de $50 \%$ nos membros superiores, seguido da coluna vertebral (39\%). Foram identificados vários aspectos de risco presentes nesta atividade profissional, tais como: altas cargas diárias de trabalho influenciando na presença da dor, alta demanda cognitiva, estresse freqüentemente presente na rotina dos ultra-sonografistas, entre outros. 0 teste de regressão demonstrou alta probabilidade de ocorrência de lesões quando o conjunto de variáveis analisadas agia concomitantemente ( $r=0,97$ ). CONCLUSÃO: De maneira geral, os resultados indicam que as atividades dos ultrasonografistas podem ser consideradas como de risco para o sistema músculo-esquelético.

Unitermos: Ultra-sonografista; Sintomas músculo-esqueléticos relacionados ao trabalho; Distúrbios osteomusculares relacionados ao trabalho.

Abstract Is ultrasonography working activity hazardous to the musculoskeletal system?

OBJECTIVE: The aim of this study was to evaluate work-related musculoskeletal symptoms, and personal and professional activities of ultrasonographist physicians. MATERIALS AND METHODS: Forty-one physicians answered a questionnaire and were interviewed regarding personal and professional activities and the occurrence of musculoskeletal symptoms. Data were analyzed using variation coefficient, Spearman $\mathbf{R}$ and Student $t$ tests. A nonlinear logistic regression equation based on the probability of discomfort was applied to evaluate the interaction among significant variables. RESULTS: The results indicated a high prevalence rate $(85 \%)$ of symptoms among physicians. Pain on the upper limbs and back pain were observed in $50 \%$ and $39 \%$ of the cases, respectively. Several risks were identified such as high daily working load influencing the presence of pain, high cognitive demand, and frequent stress in the routine activity of ultrasonographist physicians. The regression analysis showed high probability of occurrence of injuries when all variables occurred together $(r=0,97)$. CONCLUSION: Overall, the results suggest that ultrasonography working activity is a risk to the musculoskeletal system.

Key words: Ultrasonographer; Work-related musculoskeletal symptoms; Work-related musculoskeletal disorders.

\section{INTRODUÇÃO}

O número de exames realizados por técnicas sofisticadas de diagnóstico por imagem tem aumentado à medida que os

* Trabalho realizado pelo Laboratório de Fisioterapia Preven tiva e Ergonomia do Departamento de Fisioterapia da Universidade Federal de São Carlos (UFSCar), São Carlos, SP.

1. Fisioterapeuta graduada pela UFSCar, Mestre em Fisioterapia pelo Programa de Pós-Graduação em Fisioterapia da UFSCar.

2. Professora Titular do Departamento de Fisioterapia da UFSCar, Pós-doutorado em Ergonomia pela Universidade de Alberta, Canadá.

Endereço para correspondência: Letícia Holtz Barbosa. Rua Prudente de Morais, 554, ap. 84, Centro. Ribeirão Preto, SP, 14015-100. E-mail: leticiahb@zipmail.com.br

Recebido para publicação em 21/5/2003. Aceito, após revisão, em 20/8/2003. resultados desses exames tornam-se mais precisos e conduzem a diagnósticos mais confiáveis. Apesar do grande avanço tecnológico dos novos equipamentos, que permitem imagens de alta resolução, as condições ergonômicas de trabalho dos ultra-sonografistas não parecem ter acompanhado essa evolução, já que sintomas musculares têm sido fortemente associados a essa atividade ${ }^{(\mathbf{1 - 3})}$. Alguns fatores de risco para o desenvolvimento de distúrbios osteomusculares relacionados ao trabalho foram descritos para os ultra-sonografistas, tais como posturas extremas, trabalho muscular estático de alguns segmentos e movimentos altamente repetitivos, além da necessidade de aplicar pressão para segurar e manter o transdutor em posição operacional $^{(1,3,4)}$.

Vários estudos baseados em questionários revelaram altas taxas de prevalência de sintomas músculo-esqueléticos entre os ultra-sonografistas analisados. Vanderpool et $a l .{ }^{(5)}$ encaminharam um questionário para 225 ultra-sonografistas que realizavam exames do coração (ecocardiograma) e obtiveram taxa de resposta de $47 \%$, dos quais $72 \%$ eram mulheres. Dos 101 participantes, $86 \%$ relataram um ou mais sintomas físicos. Em 1996, Necas ${ }^{(6)}$ encaminhou, por correio, um questionário para 413 membros da Sociedade Médica de 
Ultra-sonografistas dos estados de Washington e Oregon, EUA. Responderam ao questionário 149 ultra-sonografistas (36\%), dos quais $81 \%$ eram sintomáticos $(66 \%)$ ou apresentavam diagnóstico de lesões por esforços repetitivos (15\%).

Um outro estudo também utilizou questionário para verificar a prevalência de desconforto presente nos profissionais que realizavam ecocardiograma ${ }^{(7)}$. Das 220 correspondências encaminhadas, 113 retornaram $(51 \%)$. Dos médicos estudados, $90(80 \%)$ relataram dor músculo-esquelética. Já o estudo realizado por Wihlidal e $\operatorname{Kumar}^{(8)}$, com ultra-sonografistas de Alberta, Canadá, apresentou taxa de resposta aos questionários de 61,5\% (96) e revelou que $88,5 \%$ dos respondentes apresentavam sintomas músculo-esqueléticos.

$\mathrm{Na}$ Itália foi realizada uma pesquisa de abrangência nacional $^{(4)}$, na qual foi encaminhado um questionário para os membros da divisão de Ultra-sonografistas da Sociedade Italiana de Radiologia e membros da Sociedade de Ultra-sonografia em Ginecologia e Obstetrícia. Dos 2.670 questionários enviados, 76,4\% foram respondidos. Entre os médicos analisados, $80 \%$ relataram sentir um ou mais sintomas relacionados ao trabalho.

Mais recentemente, foi publicado um estudo com ultra-sonografistas canadenses, no qual foram enviados por correio 232 questionários. A taxa de resposta neste estudo foi de $92 \%$. Dos médicos que participaram do estudo, $91 \%$ relataram ter sentido dores ou desconfortos desde que começaram a trabalhar com ultra-sonografia, sendo que $8 \%$ desses estavam sintomáticos no momento da pesquisa ${ }^{(\mathbf{9})}$.

Os estudos referidos anteriormente foram baseados em questionários auto-administrados e apresentaram taxas de respostas variando de $36 \%$ a $76 \%$, com exceção de Russo et al. ${ }^{(\mathbf{9})}$. Apesar do fato de questionários auto-administrados para avaliar sintomas poderem apresentar boa confiabilidade $^{(\mathbf{1 0})}$, os questionários enviados por correio são retornados apenas por parte da população endereçada. Assim, a taxa de respondentes pode apresentar viés metodológico, já que tendem a responder o questionário sobretudo aqueles indivíduos motivados a refletir sobre o assunto, que, no caso, podem ser mais sintomáticos.
Dessa maneira, uma abordagem mais direta, que inclua entrevistas e medidas objetivas de fatores de risco, parece pertinente. Ainda, cuidados adicionais na determinação da população participante são necessários. Portanto, o objetivo deste estudo foi realizar um levantamento de aspectos pessoais, ocupacionais e de sintomas músculo-esqueléticos percebidos por médicos ultra-sonografistas, por meio de questionários e de entrevistas realizadas com os participantes.

\section{MATERIAIS E MÉTODOS}

Sujeitos: Participaram deste estudo 41 médicos, sendo 15 mulheres $(36,6 \%)$ e 26 homens $(63,4 \%)$, com idade média de 37 anos (desvio-padrão: 7,1), sendo 35 sintomáticos e seis assintomáticos.

Recrutamento dos participantes: $\mathrm{O}$ recrutamento dos participantes foi realizado em algumas clínicas das cidades de Ribeirão Preto e São Carlos, SP, e Belo Horizonte, MG. Clínicas com no mínimo 70\% de médicos participantes foram incluídas no estudo, totalizando 41 médicos, que concordaram e reuniam condições mínimas para participar do estudo.

Foram também consideradas condições para participação no estudo que esses médicos realizassem exames de ultra-sonografia regularmente e que estivessem há pelo menos seis meses nessa atividade.

Materiais: Uma entrevista e um questionário com perguntas estruturadas foram aplicados a todos os participantes.

Entrevista: Utilizou-se um roteiro com 28 questões, sendo algumas adaptadas do questionário de Whilidal e $\operatorname{Kumar}^{(\mathbf{8})}$. As questões estavam divididas em quatro segmentos, relacionados aos aspectos: pessoais, do trabalho, dos sintomas músculoesqueléticos e da percepção dos sujeitos com relação aos fatores determinantes de seu desconforto. Os aspectos pessoais incluíam gênero, idade e prática de atividade física. Os sintomas músculo-esqueléticos foram caracterizados quanto à região corporal de ocorrência. Os aspectos relacionados ao trabalho eram o tempo de trabalho na profissão, o número de horas trabalhadas por dia e o número de exames realizados por dia. A percepção dos sujeitos com relação às atividades realizadas e pos- turas adotadas durante os exames de ultrasonografia foi registrada através de uma tabela adaptada de Wihlidal e Kumar ${ }^{(8)}$, que utilizava escalas subjetivas.

Questionário: Informações relacionadas ao estresse ocupacional e a aspectos organizacionais foram coletadas através de um roteiro construído para este estudo e baseado no "Occupational Stress Questionnaire" (OSQ) do Instituto de Saúde Ocupacional da Finlândia ${ }^{(11)}$. Os respondentes não foram identificados no questionário, com o objetivo de assegurar maior confiabilidade às respostas relacionadas aos aspectos ocupacionais.

O questionário era constituído de nove questões relacionadas a: percepção de tensão no trabalho (2), incluindo se o trabalho era mentalmente estressante ou distribuído de maneira desigual durante a jornada de trabalho; percepção do ambiente (2), incluindo a necessidade de se apressar para terminar o trabalho e a presença de pacientes na sala de espera em condições muitas vezes desconfortáveis (por exemplo, com bexiga cheia); percepção das sobrecargas mentais (1); relações sociais no ambiente de trabalho (2); satisfação com o trabalho (1); relação entre percepção do estresse e a presença de sintomas músculo-esqueléticos (1).

Procedimentos: As entrevistas eram conduzidas individualmente pelo mesmo entrevistador. Ao final da entrevista, os participantes responderam ao questionário. O entrevistador fornecia as orientações iniciais necessárias para o entendimento do questionário e permanecia na sala caso houvesse a necessidade de esclarecimento de alguma das questões.

Análise estatística: Realizou-se uma análise descritiva dos resultados. O teste de Levene foi utilizado para testar a homogeneidade das amostras (grupo de sujeitos sintomáticos e assintomáticos). O teste $t$ de Student comparou dados intervalares entre ambos os grupos. O coeficiente de variação foi aplicado para analisar as variações nos resultados das escalas subjetivas. Utilizou-se o coeficiente de Spearman para correlacionar o tempo gasto em cada atividade descrita e sua contribuição no desconforto percebido. Considerou-se um valor de significância de $\mathrm{p}=0,05$ para todos os testes aplicados. 
Adicionalmente, utilizou-se uma equação baseada no risco relativo para avaliar a contribuição individual das variáveis significativas na determinação dos desconfortos músculo-esqueléticos. A equação de regressão utilizada foi descrita por Gil Coury et $a l^{\left({ }^{(12)}\right.}$ e incluía as seguintes variáveis: idade, tempo de trabalho, número de exames realizados por dia, prática de atividade física, necessidade de se apressar para terminar o trabalho, distribuição desigual do trabalho durante o dia, ausência de colaboração entre os colegas e desmotivação com a atividade profissional.

\section{RESULTADOS}

Entrevista: Dos 41 participantes, 85\% (35) reportavam já ter sentido algum tipo de dor ou desconforto músculo-esquelético relacionado ao trabalho, enquanto $15 \%$ (6) eram assintomáticos.

A Figura 1 apresenta o porcentual de sintomas relatados pelos sujeitos por região corporal, na qual se observa maior concentração dos sintomas nos membros superiores, assim distribuídos: ombro $25 \%$, braços $10 \%$, cotovelos $2 \%$ antebraços $4 \%$ e punho, mãos e dedos 9\%; e nos diferentes segmentos da coluna vertebral (39\%), onde a região cervical representa 19\%, a torácica $8 \%$ e a lombar $12 \%$.

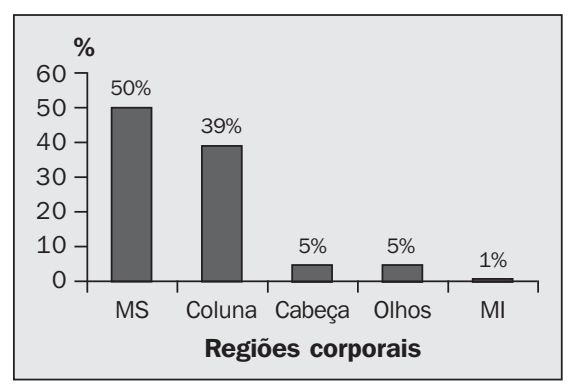

Figura 1. Porcentual dos sintomas por região corporal. (MS, membros superiores; MI, membros inferiores).

Quanto à prática de atividade física, $44 \%$ não realizavam qualquer atividade física, $41 \%$ praticavam algum tipo de exercício três vezes por semana e $15 \%$ exercitavam-se duas vezes por semana.

A Tabela 1 apresenta algumas variáveis relacionadas à exposição temporal ao trabalho, para os grupos sintomático e assintomático. Como a população revelou-se
Tabela 1 Descrição de variáveis temporais (número \pm desvio-padrão).

\begin{tabular}{|l|c|c|c|}
\hline Variáveis ocupacionais & Média sintomáticos & Média assintomáticos & Teste $t(p)$ \\
\hline Tempo de trabalho (anos) & $8,1 \pm 5,6$ & $9,0 \pm 8,6$ & 0,6301 \\
Horas trabalhadas/dia & $6,0 \pm 2,8$ & $3,9 \pm 2,2$ & 0,0029 \\
Nímero de exames/dia & $17,6 \pm 7,6$ & $9,5 \pm 5,5$ & 0,0053 \\
\hline
\end{tabular}

homogênea (Levene > 0,5), aplicou-se o teste $t$ para comparar o tempo de trabalho, número de horas trabalhadas e número de exames realizados por dia entre os sujeitos sintomáticos e assintomáticos (Tabela 1).

$\mathrm{O}$ número de horas trabalhadas e o número de exames realizados por dia apresentaram relação significativa com a presença de sintomas entre os médicos, de maneira que, quanto maior o número de exames realizados ou de horas trabalhadas, maiores as chances do médico ser sintomático. Por outro lado, o tempo de profissão não apresentou relação significativa com a presença de sintomas.

O tempo gasto nas principais atividades realizadas durante o trabalho e a contribuição de cada uma delas na presença dos desconfortos, de acordo com a percepção dos participantes, estão descritos na Tabela 2. Além disso, também são apresentados os valores do coeficiente de variação e do coeficiente de Spearman para cada uma das atividades listadas.

Os altos valores do coeficiente de variação mostrados na Tabela 2 indicam que os tos com as tarefas analisadas de forma diferente (tempo gasto), além de identificarem de maneira diversa o nível de contribuição dessas atividades para o descontrabalhadores percebem seus envolvimenforto sentido. Apesar disso, a maioria dos participantes tendeu a concordar que passavam um tempo similar (e extenso) segurando o transdutor, além de permanecer por longos períodos sentados.

O fato de o coeficiente de Spearman ser significativo para várias atividades indica uma associação positiva entre passar grande parte do tempo em cada uma dessas atividades e uma alta contribuição desta atividade para o desconforto. Apesar deste coeficiente ter-se apresentado significativo para várias atividades, apenas alguns deles apresentaram altos valores, como foi o caso de "manter o pescoço e o tronco em rotação", "torções repetitivas do pescoço e tronco" e "aplicar pressão constante no transdutor".

Questionário: Os resultados obtidos com o questionário sobre estresse ocupacional estão representados na Figura 2.

De acordo com as respostas, para 54\% dos participantes o trabalho com ultra-sonografia exige concentração intensa sempre. Também, o trabalho foi considerado, sempre ou frequientemente, mentalmente difícil para $45 \%$ dos ultra-sonografistas. A pressão decorrente do conhecimento da presença de pacientes esperando para realizarem seus exames, estando muitos deles em situações desconfortáveis, era percebida sempre ou freqüentemente por $44 \%$ dos médicos participantes. Por outro lado,
Tabela 2 Valores da média $(\bar{X})$, desvio-padrão (DP) e coeficiente de variação (CV) de uma escala variando de 0 a 5 pontos. Resultados do teste de Spearman R.

\begin{tabular}{|c|c|c|c|c|c|c|}
\hline \multirow{2}{*}{ Atividades } & \multicolumn{2}{|c|}{ Tempo gasto } & \multicolumn{3}{|c|}{$\begin{array}{c}\text { Contribuição } \\
\text { para a dor }\end{array}$} & \multirow{2}{*}{$\begin{array}{c}\text { Spearman R } \\
\mathrm{R}\end{array}$} \\
\hline & $\bar{X} \quad(D P)$ & CV & $\bar{x}$ & (DP) & CV & \\
\hline Segurar o transdutor & $4,8(0,5)$ & $10,4 \%$ & 2,9 & $(1,6)$ & $55,2 \%$ & $0,305775^{*}$ \\
\hline Aplicar pressão constante no transdutor & $2,5(0,8)$ & $32,0 \%$ & 2,8 & $(1,4)$ & $50,0 \%$ & $0,501514 *$ \\
\hline Manter o ombro em abdução & $3,7(1,0)$ & $27,0 \%$ & 4,1 & $(1,2)$ & $29,3 \%$ & $0,356997^{*}$ \\
\hline Ficar sentado por tempo prolongado & $4,8(0,4)$ & $8,3 \%$ & 1,7 & $(1,1)$ & $64,7 \%$ & $-0,206424$ \\
\hline Ficar em pé por tempo prolongado & $1,2(0,5)$ & $41,7 \%$ & 1,2 & $(0,9)$ & $75,0 \%$ & $0,313489 *$ \\
\hline Manter pescoço e tronco em rotação & $2,7(1,4)$ & $51,9 \%$ & 2,6 & $(1,4)$ & $53,8 \%$ & $0,732520^{*}$ \\
\hline Torções repetitivas do pescoço e tronco & $2,6(1,3)$ & $50,0 \%$ & 2,1 & $(1,3)$ & $61,9 \%$ & $0,620166 *$ \\
\hline Levantar/ajudar o paciente & $1,5(0,9)$ & $60,0 \%$ & 1,1 & $(0,3)$ & $27,3 \%$ & $0,357765^{*}$ \\
\hline Manipular o "track-ball” & $3,5(1,1)$ & $43,0 \%$ & 1,2 & $(0,6)$ & $50,0 \%$ & 0,062823 \\
\hline Digitar no teclado & $2,8(1,1)$ & $39,3 \%$ & 1,5 & $(0,9)$ & $60,0 \%$ & $0,390928 *$ \\
\hline
\end{tabular}




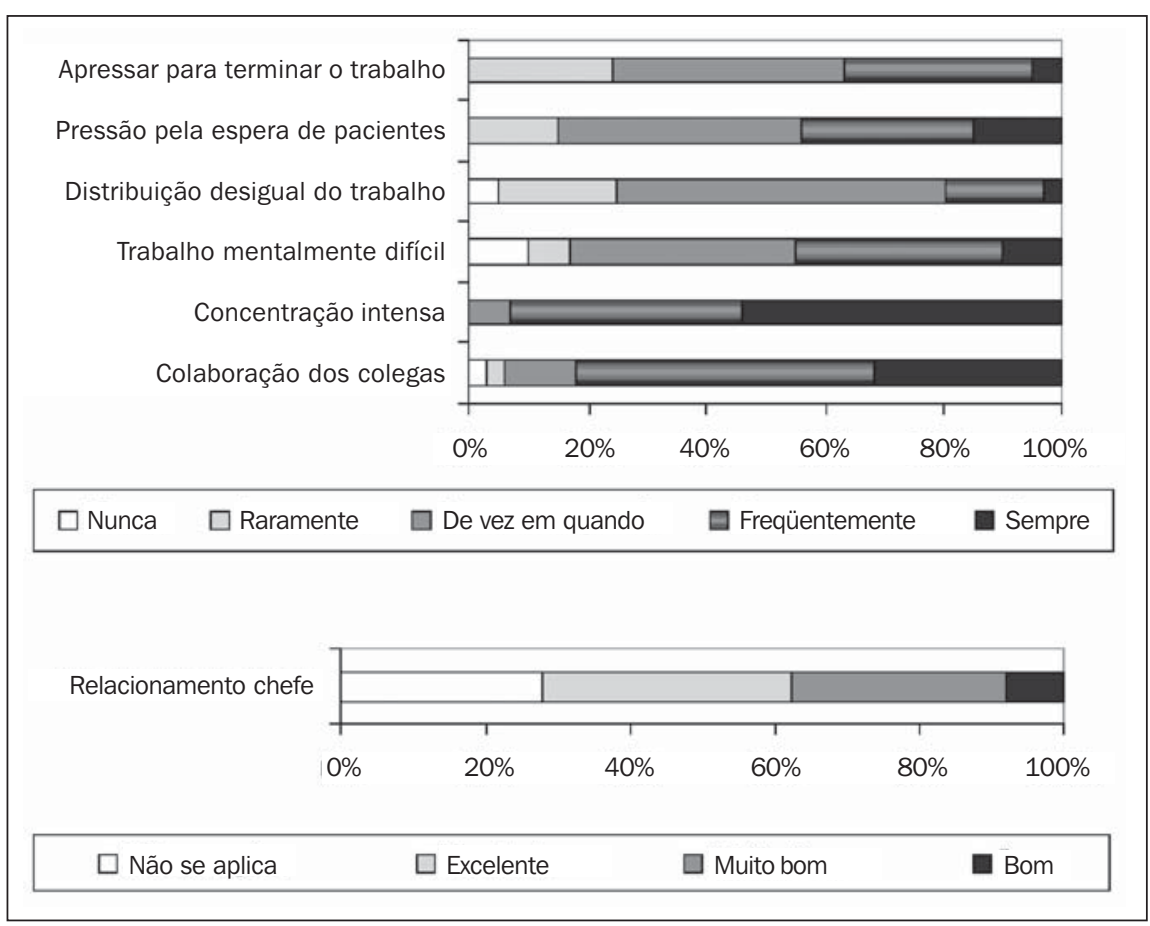

Figura 2. Representação de algumas das questões relacionadas ao estresse ocupacional percebido pelos médicos ultra-sonografistas.

as relações sociais relacionadas ao apoio de superiores e à colaboração dos colegas de trabalho foram relatadas como positivas.

Na Figura 2 é possível identificar que $37 \%$ dos sujeitos percebiam a necessidade de se apressarem para realizar o trabalho, com freqüência moderada ou intensa. Ainda com relação à distribuição da quantidade de trabalho ao longo da jornada, em $56 \%$ dos casos ela ocorria de maneira desigual, de vez em quando, ao passo que para $20 \%$ dos sujeitos esta distribuição ocorria de maneira desigual, frequientemente ou sempre.

A satisfação com o trabalho e a possível relação entre diferentes estados de ânimos e a presença ou aumento do desconforto sentido também foram analisadas. Vários participantes relataram estar motivados com o trabalho que desenvolviam (68\%), no entanto, $73 \%$ dos médicos percebiam uma relação entre os estados de ânimo percebidos e a presença de desconfortos.

Os resultados da análise de regressão mostraram que médicos que trabalhavam sob as condições adversas, avaliadas no presente estudo, apresentavam alta probabilidade $(r=0,97)$ de apresentar desconfortos ou dores músculo-esqueléticas. Apesar disso, a contribuição individual de cada variável para a determinação do desconforto foi baixa. As variáveis que apresentaram as maiores contribuições foram "necessidade de se apressar para terminar o trabalho" (10\%) e "ausência de colaboração entre os colegas” $(8,1 \%)$. Todas as outras variáveis apresentaram valores baixos, o que sugere que suas principais contribuições ocorreram na interação entre elas, ou seja, a ocorrência simultânea destas variáveis parece potencializar o efeito individual de cada uma.

\section{DISCUSSÃO}

Os resultados referentes aos aspectos subjetivos, como sintomas relatados, percepção da contribuição das atividades para os desconfortos e estresse ocupacional, indicam que a atividade do ultra-sonografista apresenta claros riscos para o sistema músculo-esquelético.

No que diz respeito aos desconfortos relatados, $85 \%$ dos médicos indicaram ter um ou mais sintomas músculo-esqueléticos relacionados ao trabalho. Este porcentual condiz com valores encontrados na literatura, em que a taxa de incidência de desconfortos ou lesões músculo-esqueléticos variou de $80 \%$ a $88,5 \%{ }^{(4-9)}$.
Dentre as regiões corporais mais acometidas nos sujeitos sintomáticos estão os membros superiores ( $50 \%$ das queixas) e a coluna vertebral (39\% das queixas). Estas regiões foram também identificadas como relevantes em outros estudos ${ }^{(6-9)}$. Aparentemente, o desconforto ou dor nessas regiões são decorrentes das posturas adotadas por esses profissionais durante a realização dos exames. Segundo Magnavita et al. ${ }^{(4)}$, na maior parte do tempo os ultra-sonografistas mantêm o braço que segura o transdutor em abdução e sem apoio, e a coluna cervical fletida e rodada. Além disso, ocorre uma contração isométrica dos músculos do pescoço, coluna e membro superior, visando à estabilização do braço, de maneira a permitir que movimentos precisos das mãos sejam realizados. A manutenção dessa postura estática dos segmentos mais proximais requer consideráveis níveis de contração muscular isométrica, o que predispõe rapidamente a fadiga e desconfortos.

Na mesma direção, os médicos perceberam uma correlação significativa entre as atividades ou os movimentos realizados e a ocorrência de dor no segmento corporal relacionado com a atividade, principalmente para as posturas de pescoço e preensão do transdutor. A associação positiva entre essas variáveis - atividade e dor reforça o papel promocional dos aspectos físicos do trabalho na ocorrência dos desconfortos.

No que se refere aos aspectos temporais, a duração de cada exame parece depender de aspectos variados, tais como dificuldade em localizar as alterações investigadas, condições do paciente como obesidade, etc. Portanto, torna-se difícil estimar precisamente quanto tempo cada exame durará. $\mathrm{O}$ fato de surgirem exames inesperados e urgentes também acaba influenciando a agenda de atendimentos dos médicos, tornando a distribuição dos exames desigual ao longo do dia ou da semana e provocando um acúmulo de pacientes na sala de espera. A percepção dos atrasos e o acúmulo de pessoas na sala de espera, associados à necessidade de intensa concentração para evitar erros e acrescidos do fato de terem que tomar decisões rápidas, as quais podem ter conseqüências sérias para a saúde dos pacientes, pode tornar essa 
atividade tensa e envolver alta demanda cognitiva.

Westgaard ${ }^{(\mathbf{1 3})}$ relatou vários estudos associando fatores psicossociais e psicológicos negativos com a tendência de desenvolver tensões musculares e sintomas. De acordo com esse autor, condições psicossociais desfavoráveis no trabalho podem iniciar ativações musculares não voluntárias. Ainda neste sentido, Waersted et al. ${ }^{(\mathbf{1 4})}$ analisaram as respostas eletromiográficas do músculo trapézio quando os sujeitos realizavam um teste com e sem incentivo financeiro. Nos testes realizados com incentivo financeiro havia um aumento significativo na tensão muscular quando comparado à atividade muscular encontrada na tarefa sem incentivo. Apesar do fato de que, no presente estudo, a tensão não ter resultado positivo (dinheiro), mas sim negativo (apreensão e necessidade de se apressar devido a espera de pacientes), os efeitos nos médicos parecem ter sido igualmente desfavoráveis.

Uma outra possibilidade para a ativação muscular não voluntária é a atividade muscular contínua exercida com a finalidade de estabilizar a cabeça, ombros e braços, visando permitir os movimentos dos punhos e mãos. Esta ativação muscular contínua gera mudanças metabólicas locais que, de acordo com Johansson e Sojka ${ }^{(15)}$ podem levar a um padrão reflexo de propagação. Por meio deste mecanismo, à medida que as fibras começam a se tornar fatigadas, novas fibras são ativadas reflexamente, que também serão sobrecarregadas em decorrência da alta demanda física do trabalho. Assim, é bastante possível que diferentes mecanismos, fisiológicos e psicossociais, atuem associadamente na explicação da alta prevalência de sintomas nos ultra-sonografistas.

No que diz respeito às variáveis envolvendo tempo de exposição ao trabalho (anos de profissão, horas de trabalho e número de exames/dia), apesar de o tempo de profissão ter apresentado relação com a presença dos sintomas, o número de horas trabalhadas e o número de exames realizados por dia mostraram associação significativa. Estudos investigando diferentes atividades têm reportado associações positivas entre horas trabalhadas por dia e desconfortos ou lesões. Em um estudo rea- lizado por Fransson-Hall et al. ${ }^{(\mathbf{1 6})}$, em que foram analisados trabalhadores de linha de montagem de automóveis, encontrou-se associação entre o maior tempo de exposição a fatores de risco e a presença de sintomas músculo-esqueléticos. Margolis e Kraus $^{(17)}$, ao estudarem trabalhadores em caixas de supermercados, observaram que a taxa de prevalência estava relacionada, entre outros fatores, com a média de horas trabalhadas por semana. O mesmo tipo de associação também foi encontrado por Morgenstern et al. ${ }^{(\mathbf{1 8})}$ ao analisarem mulheres que trabalhavam como caixas de mercearias. No presente estudo, os efeitos da exposição ao trabalho foram melhor expressos por horas trabalhadas por dia, do que anos trabalhados.

$\mathrm{O}$ pequeno tamanho da amostra limitou o entendimento de algumas relações que, possivelmente, poderiam esclarecer melhor a complexa determinação dos sintomas e lesões músculo-esqueléticas relacionadas ao trabalho, nos ultra-sonografistas. Entretanto, a obtenção de grandes amostras pode ser muito difícil ao se conduzir estudos nos quais algumas condições metodológicas são estabelecidas, tais como $70 \%$ de taxa de participação em cada clínica/setor de trabalho e a adoção de uma abordagem mais direta (entrevistas), como no presente estudo.

Por último, de acordo com os resultados obtidos, as atividades dos ultra-sonografistas podem ser consideradas como de risco para o sistema músculo-esquelético, devido à alta prevalência de sintomas identificados e também às demandas psicológicas, cognitivas e físicas percebidas. Dessa maneira, os resultados deste estudo podem ser vistos como um alerta para os médicos ultra-sonografistas e sugerem a necessidade de ações voltadas para a prevenção dos aspectos ocupacionais críticos aqui relatados.

\section{Agradecimentos}

Agradecemos aos médicos que colaboraram com este estudo, especialmente aqueles que atenciosamente abriram suas clínicas de maneira que este estudo pudesse ser realizado, e ao apoio do CNPq (Proc. no 550487/2002-6), Capes e Colégio Brasileiro de Radiologia e Diagnóstico por Imagem (CBR).

\section{REFERÊNCIAS}

1. Craig M. Sonography: an occupational health hazard? J Diag Med Sonogr 1985;3:121-6.

2. Craig M. Occupational hazards of sonography: an update. J Diag Med Sonogr 1990;1:47-50.

3. Mercer RB, Marcella CP, Carney DK, McDonald RW. Occupational health hazards to the ultrasonographer and their possible prevention. J Am Soc Echocardiogr 1997;10:363-6.

4. Magnavita N, Bevilacqua L, Mirk P, Fileni A, Castellino N. Work-related musculoskeletal complaints in sonologists. J Occup Environ Med 1999;41: 981-8.

5. Vanderpool HE, Friis EA, Smith BS, Harms KL. Prevalence of carpal tunnel syndrome and other work-related musculoskeletal problems in cardiac sonographers. J Occup Med 1993;35:604-10.

6. Necas M. Musculoskeletal symptomatology and repetitive strain injuries in diagnostic medical sonographers: a pilot study in Washington and Oregon. J Diag Med Sonogr 1996;12:266-73.

7. Smith AC, Wolf JG, Xie GY, Smith MD. Musculoskeletal pain in cardiac ultrasonographers: results of a random survey. J Am Soc Echocardiogr 1997; 10:357-62.

8. Wihlidal LM, Kumar S. An injury profile of practicing diagnostic medical sonographers in Alberta. International Journal of Industrial Ergonomics 1997;19:205-16

9. Russo A, Murphy C, Lessoway V, Berkowitz J. The prevalence of musculoskeletal symptoms among British Columbia sonographers. Appl Ergon 2002; 33:385-93.

10. Booth-Jones AD, Lemasters GK, Succop P, Atterbury MR, Bhattacharya A. Reability of questionnaire information measuring musculoskeletal symptoms and work histories. Am Ind Hyg Assoc J 1998;59:20-4.

11. Elo AL, Leppanen A, Lindstrom K, Ropponen T. Occupational stress questionnaire: user's instructions (OSQ). Helsinki, Finland: Institute of Occupational Health, 1992.

12. Gil Coury HJC, Porcatti IA, Alem MR. Influence of gender on work-related musculoskeletal disorders in repetitive tasks. International Journal of Industrial Ergonomics 2002;29:33-9.

13. Westgaard RH. Effects of psychosocial demand and stress on neuromuscular function. In: Moon SD, Sauter SL, eds. Beyond biomechanics: psychosocial aspects of musculoskeletal disorders in office work. London: Taylor \& Francis, 1996:75-89.

14. Waersted M, Bjorklund RA, Westgaard RH. The effect of motivation on shoulder-muscle tension in attention-demanding tasks. Ergonomics 1994;37: 363-76.

15. Johansson H, Sojka P. Pathophysiological mechanisms involved in genesis and spread of muscular tension in occupational muscle pain and in chronic musculoskeletal pain syndromes: a hypothesis. Med Hypotheses 1991;35:196-203.

16. Fransson-Hall C, Bystrom S, Kilbom A. Self-reported physical exposure and musculoskeletal symptoms of the forearm-hand among automobile assembly-line workers. J Occup Environ Med 1995; 37:1136-44.

17. Margolis W, Kraus JF. The prevalence of carpal tunnel syndrome symptoms in female supermarket checkers. J Occup Med 1987;29:953-6.

18. Morgenstern H, Kelsh M, Kraus J, Margolis W. A cross-sectional study of hand/wrist symptoms in female grocery checkers. Am J Ind Med 1991;20: 209-18. 